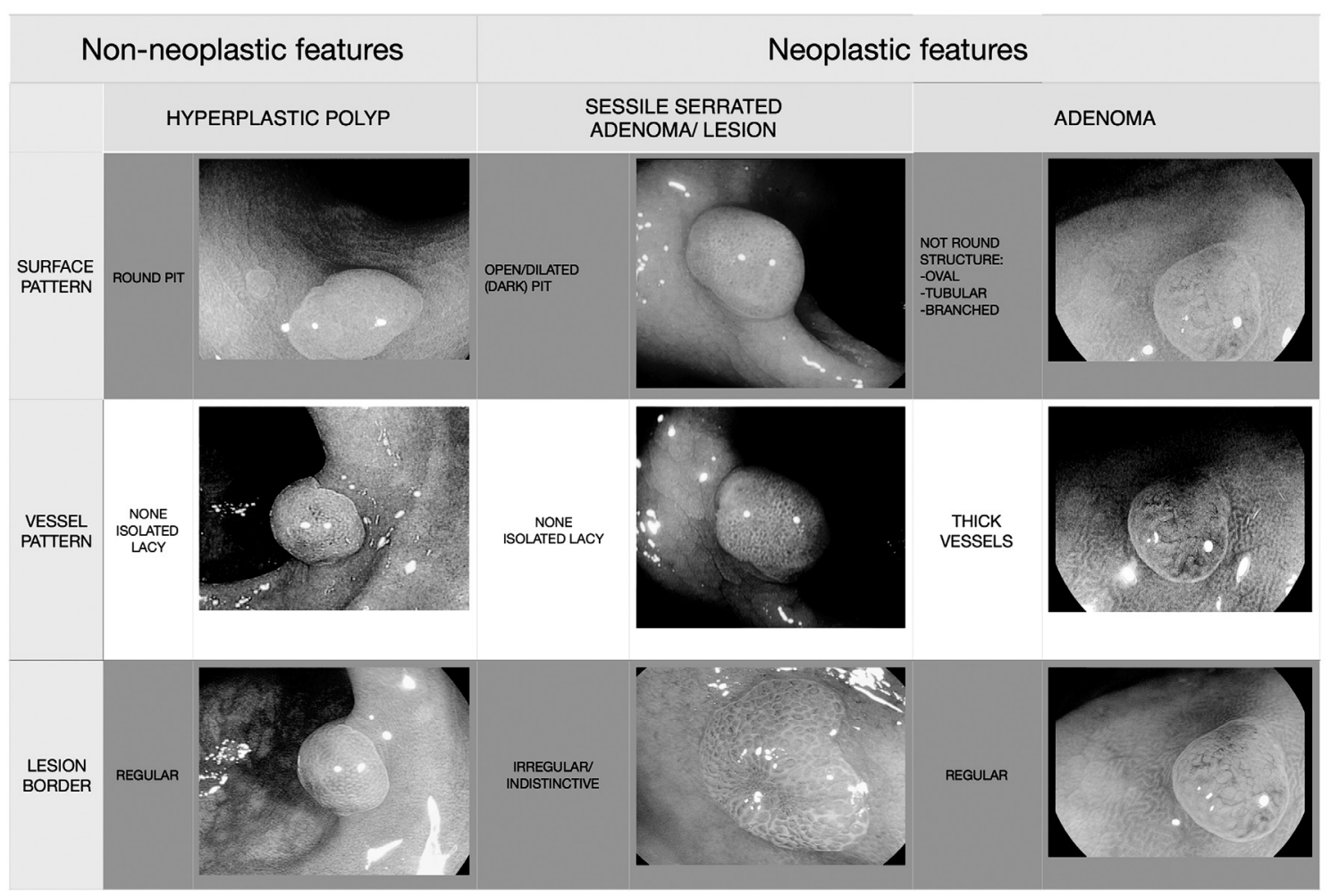

Abstract ADWE-01 Figure 1

Introduction Experts are able to predict and differentiate between neoplastic and non-neoplastic colonic polyps with high accuracy and meet the PIVI (Preservation and Incorporation of Valuable Endoscopic Innovations) thresholds. However, this has not been replicated in non-experts and cannot be currently recommended in clinical practice. The aim of this randomised trial was to establish the optimum method of training to increase the diagnostic accuracy of diminutive/ small colonic polyp histological prediction by trainee gastroenterologists.

Methods Gastroenterology trainees from the Midlands, UK, reviewed 78 videos (48 i-Scan-OE $+30 \mathrm{NBI}$ ) of diminutive/ small polyps. Participants were randomised to computer-based learning or didactic training. The 78 videos (randomised order) were re-assessed. The NICE (NBI International Colorectal Endoscopic) classification and SIMPLE (Simplified Identification Method for Polyp Labelling during Endoscopy) classification systems were used to classify diminutive/small polyps (figure 1).

Results 16 trainees (12 gastroenterology trainees and 4 naïve endoscopists) were randomised to receive either self-training $(n=8)$ or didactic training $(n=8)$. A higher proportion of high confidence predictions of polyps were made by the self-training group vs didactic group when using the SIMPLE classification $77.1 \%$ vs. $69.9 \% \quad(\mathrm{p}<0.005)$ and using the NICE classification $77 \%$ vs. $69.8 \% \quad(p=0.006)$. When using NICE classification, the sensitivity of the self-training group was $72 \%$ vs. $83 \% \mathrm{p}<0.0005$, and the accuracy $66.1 \%$ vs. $69.1 \%$. When using SIMPLE classification the sensitivity was $78 \%$ vs. $83 \%$ (NS) and accuracy $65.7 \%$ vs. 69\% (table 1).
Abstract ADWE-01 Table 1 Diagnostic performance to predict colonic polyp histology

\begin{tabular}{|c|c|c|c|c|}
\hline & & Self-training $[95 \% \mathrm{Cl}]$ & Didactic $[95 \% \mathrm{Cl}]$ & P-value \\
\hline & Sensitivity & $0.78[0.73-0.82]$ & $0.83[0.78-0.86]$ & 0.148 \\
\hline \multirow[t]{3}{*}{ SIMPLE } & Specificity & $0.51[0.48-0.57]$ & $0.52[0.46-0.58]$ & 0.735 \\
\hline & Accuracy & $0.66[0.62-0.69]$ & $0.69[0.65-0.73]$ & 0.225 \\
\hline & Sensitivity & $0.72[0.69-0.77]$ & $0.83[0.79-0.87]$ & 0.0005 \\
\hline \multirow[t]{2}{*}{ NICE } & Specificity & $0.59[0.53-0.65]$ & $0.52[0.46-0.58]$ & 0.106 \\
\hline & Accuracy & $0.66[0.62-0.70]$ & $0.69[0.65-0.73]$ & 0.275 \\
\hline
\end{tabular}

Conclusions Self-learning for the prediction of diminutive/small polyp histology is a method of training that can achieve results similar to the more labour intensive and expensive didactic training. The availability of adequate self-learning teaching modules that teach how to differentiate neoplastic vs non-neoplastic colonic polyps with high accuracy could enable more widespread implementation of optical diagnosis in clinical practice.

\section{ADWE-02 DIAGNOSTIC YIELD AND EARLY DISCHARGE IN GASTROINTESTINAL BLEEDING: COMPARATIVE TRIAL BETWEEN CAPSULE ENDOSCOPY AND GASTROSCOPY}

${ }^{1}$ Hey-long Ching*, ${ }^{1}$ Melissa F Hale, ${ }^{1}$ Reena Sidhu, ${ }^{2}$ Sabina Beg, ${ }^{2}$ Krish Ragunath, ${ }^{1}$ Mark E McAlindon. 'Royal Hallamshire Hospital, Sheffield, UK; ${ }^{2}$ Nottingham University Hospitals, Nottingham, UK

10.1136/gutjnl-2018-BSGAbstracts. 10 
Introduction Capsule endoscopy may have a role in triaging patients with suspected acute upper gastrointestinal (GI) bleeding who need further invasive investigation.

Method The Mirocam Navi (Intromedic Ltd., Korea) is a single camera capsule endoscope steerable by a handheld magnet. Magnetically assisted capsule endoscopy (MACE) was performed in stable patients with suspected acute upper GI bleeding and a decision made as to whether same day discharge was appropriate, prior to gastroscopy (OGD) performed by a different, blinded endoscopist followed by standard care by the admitting team during which small bowel capsule endoscopy was completed. Diagnostic yield and comfort were compared. Potential impact on hospital stay was examined and MACE mucosal visualisation quality was assessed.

Results A total of 90 upper GI lesions were detected in 30 patients $(80 \%$ male, median age 57 years $(\mathrm{IQR}=26)): 21$ (23\%) concomitantly identified by MACE and OGD, 49 $(54 \%)$ by MACE alone and $20(22 \%)$ by OGD alone (CI $0.05-0.58 ; \mathrm{p}=0.0007)$. MACE and OGD concomitantly detected $50 \%$ of major pathologies (gastric and duodenal ulcers and oesophageal and gastric varices $(n=2$ each)). MACE alone identified 31\% (D1 ulcers $(n=4)$ and varices $(n=1))$ whereas OGD alone identified three D1 ulcers $(19 \% ; \mathrm{p}=0.7)$. Ulcers (all classified as Forest class III at OGD) missed by MACE were due to rapid D1 transit and insufficient imaging. Review of small bowel images identified additional causes for GI bleeding distal to D2 in five cases: small bowel lymphoma $(n=1)$, angioectasia $(n=2)$, fresh bleeding in the proximal and distal small bowel with no culprit lesion seen $(n=1$ for each). MACE correctly suggested safe discharge for 21/21 patients. The median hospital stay of uncomplicated admissions where MACE suggested discharge was 53 (range 14-103) hours. Pain, discomfort and distress scores were lower with MACE than OGD $(\mathrm{p}<0.05)$. Median MACE visualisation scores (worst-best:1-5) were excellent in the cardia (5), gastric body (5), antrum (5) and pylorus (5), good in the oesophagus (4) and fundus (3) but poor in D1 (0) and D2 (0): Kruskal-Wallis $\mathrm{H}$ test, $\chi^{2}=133 \quad(\mathrm{p}<0.05)$, lowest rank visualisation score in D1.

Conclusions MACE has better diagnostic yield than OGD in suspected upper GI bleeding (with both modalities missing lesions), is better tolerated and used 'at the front door' might allow immediate discharge for two thirds of patients saving a median hospital stay for each of over two days. In addition, it suggests that about $10 \%$ of suspected upper GI bleeding may have a small bowel source.

\section{ADWE-03 CRYOABLATION FOR THE TREATMENT OF REFRACTORY OESOPHAGEAL NEOPLASIA AFTER UNSUCCESSFUL FIRST LINE ENDOSCOPIC ERADICATION THERAPY}

${ }^{1}$ Durayd Alzoubaidi*, ${ }^{2}$ Cormac Magee, ${ }^{2}$ Martin Everson, ${ }^{2}$ David Graham, 'Laurence Lovat,
${ }^{1,2}$ Rehan Haidry. ${ }^{1} U C L$, London, UK; ${ }^{2} U C L H$, London, UK 10.1136/gutjnl-2018-BSGAbstracts.11

Introduction Endoscopic eradication therapy EET with endoscopic mucosal resection EMR for visible lesions followed by Radiofrequency ablation RFA for flat Barrett's oesophagus BE is the gold standard treatment for patients with $\mathrm{BE}$ related neoplasia. In a minority of patients EET is unsuccessful and alternative therapies are desirable. Cryoablation with the Cryoballoon device (C2 therapeutics) is a novel ablative therapy that uses cycles of freezing and thawing to induce cell death by intra and extracellular ice formation, vascular injury, and apoptosis.

Aim Single centre study to evaluate the feasibility of the focal cryoablation device for the treatment of areas of refractory oesophageal neoplasia in patients who had undergone first line EET. Endpoints: CR-D and CR-IM at 3 month follow up. The rate of stenosis and adverse events were also studied.

Method A total of 16 cases (11 M,5 F) have been treated by a single experienced endoscopist. Baseline histology included 7 Low Grade Dysplasia LGD, 5 High Grade Dysplasia HGD, 2 Intramucosal cancer (IMC) and 2 patients with Early Squamous Cell Neoplasia ESCN. All patients had residual neoplasia after sequential first line EET. The median length of dysplastic $\mathrm{BE}$ treated was $3 \mathrm{~cm}$ (Range: 1-10) in 14 cases and $3 \mathrm{~cm}$ of a visible lugols-voiding-mucosa in 2 patients with ESCN. An average of 10 ablations applied per patient (range 2-24). Each selected area of visible dysplasia received $10 \mathrm{~s}$ of ablation. Only 1 session of cryoablation per patient. Endoscopy and rebiopsy from the treated sites were taken at 3 months post ablation to assess for end points.

Results CR-D achieved in 75\% (12/16) of all patients at first follow up. In those with BE the CR-D was $79 \%(11 / 14)$ and CR-IM 36\% (5/14). 1 of the patients with ESCN did not respond to Cryoablation and was referred for radiotherapy. There was progression from LGD to HGD in $1 \mathrm{BE}$ case that was treated with EMR. Technical difficulty due to challenging anatomy was noted in 1 case with tortuous and dilated oesophagus. There were no device malfunction or adverse events. Stenosis was noted in $6 \%$ of cases.

Abstract ADWE-03 Table 1
\begin{tabular}{|c|c|}
\hline $\begin{array}{c}\text { Technical difficulty due to } \\
\text { Anatomy }\end{array}$ & $\begin{array}{c}6 \%(1 / 16) \\
\text { tortuous and dilated oesophagus }\end{array}$ \\
\hline $\begin{array}{c}\text { Technical difficulty due to } \\
\text { the Cryoablation Device }\end{array}$ & 0 \\
\hline Stenosis & $6 \%(1 / 16)$ \\
\hline No response & $13 \%(2 / 16)$ \\
\hline Progression & (Progressed from LGD to HGD) \\
\hline CR-D & $75 \%(12 / 16)$ \\
\hline CR-IM & $36 \%(5 / 14)$ \\
\hline
\end{tabular}

Conclusion In this single centre feasibility cohort study, Cryoablation with the cryoballoon device appears to be a viable strategy in patients with refractory neoplasia after sequential first line EET. It is well tolerated and partially successful in obtaining CR-D and CR-IM in 'treatment-refractory' patients with BE dysplasia. Further trials of dosimetry, efficacy and safety in 'treatment-naïve' patients with randomised controlled trials is recommended and are underway. 